Making and processing a referral using eC: Once a referral has been made in PC, it appears on-line and a hepatology secretary logs the referral, opening the episode of care and informs the designated hepatologist that a referral has been received. The eC takes approximately $15 \mathrm{~min}$ of consultant time to complete but varies depending on case complexity. Once completed, the hepatologist informs the secretary and they log a 'completed episode of care' ensuring the trust is paid for the clinical encounter. Referrals are audited on a 6 monthly basis.

Results Between March 2012 - Oct 2013, 81 eC were completed (12 in months 1-6, 16 in months 7-12, 40 in months 13-18, 13 in months 19-20). A SC appointment was avoided in $78 \%$ of patients $(n=63)$ resulting in a cost saving to PC of $£ 16,443[63 \times \mathrm{eC}(£ 23)=1,449$ vs $63 \times$ new patient referrals $(£ 181)=£ 11,403+1 \mathrm{x}$ follow up/patientn $(£ 103)=£ 6489)$. Median response time for $\mathrm{eC}$ was 2 days, $43 \%$ were completed within the same working day.

Conclusion Hepatology $\mathrm{eC}$ is beneficial for patient care, with specialist advice being provided within one working day in a substantial number of cases, and is clearly cost effective, making eC popular with PC. However, until a more slim-line IT system is developed reducing the number of steps involved in completing an $\mathrm{eC}$, and the cost per eC increased, it appears to be beneficial for all parties except SC.

Disclosure of Interest None Declared.

\section{PTH-068 REDUCING THE OUTPATIENT BURDEN OF 2 WEEK WAIT UPPER GI REFERRALS}

S Singh*, H Dhaliwal, R Keld. Gastroenterology, Wrightington, Wigan and Leigh NHS Trust, Wigan, UK

\subsection{6/gutjnl-2014-307263.514}

Introduction The two week wait (2WW) referral leads to significant burden on outpatient clinics. This delays appointments for patients that may not fit the $2 \mathrm{WW}$ criteria. Only $5 \%$ of patients referred as $2 \mathrm{WW}$ will have an upper GI malignancy and it may not be necessary for all these patients to be reviewed urgently in clinic. Previously at our hospital, most upper GI 2WW referrals were booked an OGD (performed by any available endoscopist) in addition to a clinic appointment on receipt of the referral. In order to streamline the service, in January 2013 patients were triaged to either an OGD or a clinic appointment. The index OGD's are now done on consultant's list (Gastroenterologist and Upper GI Surgeon) with a clinical assessment at their OGD appointment. Further management is protocol based and dependant upon the assessment and OGD findings. The aim of this study was to determine if this change in practice is effective and safe.

Methods Patients referred as a 2WW in January and February 2012 were compared to those referred in January, February, August and September 2013. Only patients triaged directly to OGD were included (77/143 (54\%) in 2012 and 180/291 (62\%) in 2013). 14 patients were excluded from further analysis due to non-attendance.

Results Total cancer detection for all referrals was 8\% in 2012 and $9 \%$ in 2013. In patients selected for a direct OGD referral, 7 upper GI cancers were diagnosed in 2012 and 14 in 2013. After the OGD, $4(5 \%)$ patients in 2012 were immediately discharged back to the GP, compared to $33(20 \%)$ in 2013 ( $\mathrm{p}=$ 0.003). Of those attending clinic post OGD, 9 patients $(13 \%)$ were given a routine appointment in 2012 compared to 50 $(37 \%)$ in $2013(\mathrm{p}=0.0002)$. Comparing the two years, there was a $32 \%$ reduction in the requirement of urgent outpatient appointments (83\% had urgent OPD in 2012 compared to 51\% in 2013, p $=0.0001)$. Of those discharged in 2013, 85\% had documentation of the current symptoms at time of OGD and in $94 \%$, treatment advice was provided to the GP. One patient was discharged after an OGD showing grade B oesophagitis and symptom improvement with PPI. Unfortunately, a re-referral 8 weeks later for worsening symptoms found oesophageal cancer on OGD.

Conclusion The introduction of consultant assessment as a first contact for all OGD 2WW referrals has led to a significant reduction in the requirement of urgent outpatient clinic appointments by one third. Waiting times for all clinic referrals have reduced significantly, amounting to $54 \%$ reduction in the number of patients waiting more than 9 weeks for a first appointment. Cancer detection is comparable to the previous model of care. Patients with ongoing symptoms at the time of endoscopy need follow up. In hindsight the missed cancer should have had an oesophageal biopsy, but this is clinical judgement and we do not believe the new service accounted for this delay.

Disclosure of Interest None Declared.

\section{PTH-069 NURSE TELEPHONE TRIAGED STRAIGHT TO TEST COLONOSCOPY}

${ }^{1}$ A Thapar, 'S Rodney*, 'D Haboubi, '1 Wilson, ${ }^{1} \mathrm{C}$ Bhan, ${ }^{1} \mathrm{M}$ Walshe, ${ }^{2} \mathrm{~J}$ Haddow, ${ }^{1}$ A Oshowo, ${ }^{1} \mathrm{H}$ Mukhtar. ${ }^{1}$ Surgery, Whittington Hospital, London, UK; ${ }^{2}$ National Centre for Bowel Research and Surgical Innovation, Queen Mary University of London, London, UK

\subsection{6/gutjnl-2014-307263.515}

Introduction In 2011 patients referred with suspected colorectal cancer at our institution waited a median of 36 days (IQR2846) for a treatment plan. This resulted in $61 \%$ of colorectal cancerstaking over 31 days to reach a decision to treat. We aimed to reduce the time spent in the diagnostic phase, which was perceived to be the main hold up in the fast track pathway.

Methods A quality improvement approach was employed to change the new pathway from the existing clinic-first service to a straight-to-test service. The new nurse-led telephone triage service confirmed symptoms and assessed fitness for colonoscopy, with higher-risk patients defaulting to flexible sigmoidoscopy or clinic. Results for the first year of the new service are presented.

Results 438 patients were referred between 1/10/2012 and 1/10/ 2013. $222(50 \%)$ went straight to colonoscopy and $136(31 \%)$ to flexible sigmoidoscopy, 46 (11\%) went to clinic, $32(7 \%)$ patients did not attend and data was missing for $2(1 \%)$ patients. Final diagnoses are shown in the attached figure. Colorectal cancer was found in 14/358 patients (4\%). Median time from receipt of referral to first endoscopy was 13 days (IQR 11-20), with 128/348 patients (29\%) waiting more than 14 days. Median time to decision to treat colorectal cancer was 25 days (IQR 20-34) in straight to test patients, a significant reduction compared to 2010-2011 ( $\mathrm{p}=0.01)$, with $5 / 14(36 \%)$ waiting more than 31 days. Median time to first oncological treatment was 40 days (IQR 28-44), with 1/14 (7\%) waiting more than 62 days. 41/66 (62\%) of patients with a normal colonoscopy were discharged directly from endoscopy back to their GP.

Conclusion The new straight to test service was applicable to the majority of new colorectal fast track patients and a high patient uptake was observed. Colorectal cancer was in fact uncommon, which is being fed back to those referring into the 


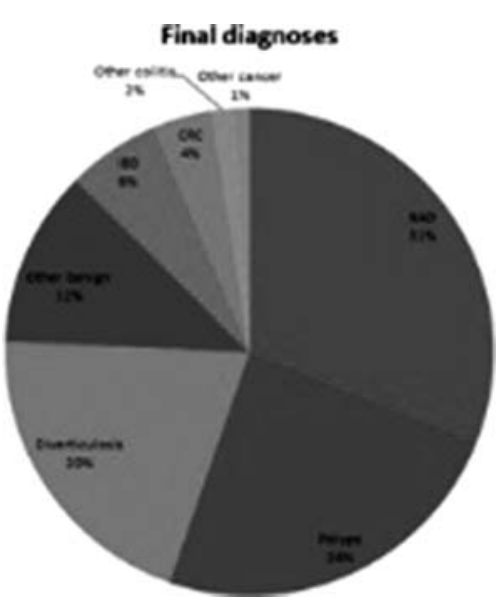

Abstract PTH-069 Figure 1

system. Physical colorectal clinic appointments were saved in four-fifths of new patients and in two-thirds of those with a normal colonoscopy, which could be allocated to newly diagnosed cancers, or those requiring treatment for benign conditions. The straight to endoscopy service resulted in an average reduction of 11 days in making a treatment plan for new colorectal cancers. This contributed towards a low rate of breaches of the 62 day treatment target. However one-third of new cancer patients still waited over a month for a decision to treat, highlighting the extra time required for ancillary investigations and MDT discussion. These can be addressed by triggering staging investigations and MDT discussion at the time of endoscopy.

Disclosure of Interest None Declared.

\section{PTH-070 NURSE LED ONE STOP UPPER GI CLINICS ARE SAFE AND ALLOW RAPID ASSESSMENT OF PATIENTS WITH SUSPECTED GASTRO-OESOPHAGEAL MALIGNANCY}

S Williams*, B Ashall, G Cave, A Bassi, PK Flanagan. Gastroenterology, Whiston Hospital, Whiston, UK

\subsection{6/gutjnl-2014-307263.516}

Introduction Meeting 2 week referral targets presents a challenge to many hospitals. Commonly patients are seen in clinic prior to investigation with resultant additional delays in time to investigation. Nurse led one stop clinics where patients undergo clinical assessment and endoscopic and/or radiological assessment on the same day have the potential to shorten time to investigation, allow rapid complete clinical assessment and meet the demand for 2 week wait (2WW) referrals.

Methods

Aims

To assess the efficacy and safety of a nurse led one stop suspected upper GI cancer clinic in meeting 2 week targets. To determine outcomes for patients referred on a $2 \mathrm{WW}$ pathway.

Methods All patients referred to Whiston Hospital on a two week upper GI pathway within the 6 month period from November 2012 to April 2013 were assessed. Additionally all upper GI cancers diagnosed in the same period were separately analysed. Patients were identified using hospital IT systems and data collated on demographics, referring symptoms, investigations and patient outcomes. Analysis was performed using StatsDirect v2.6.8.

Results Complete data was available for 202 patients (61\%). $40 \%$ of patients did not meet criteria for 2 week referral. One stop clinics enabled complete assessment and investigation of patients within 2 weeks (mean $11.6 \pm 0.63$ days). Time to first investigation was significantly quicker than patients seen in clinic (11.6 vs 18 days, $\mathrm{p}<0.005$, ANOVA) and was no different than open access endoscopy (11 days, $\mathrm{p}=0.96)$. Cancers were identified in 15 (7.4\%), of which only 8 (57\%) were upper GI. No significant differences in patient outcome (time to investigation/pathology identified) were seen between nurse led and physician led clinics. In the same time period 53 upper GI cancers were diagnosed meaning only $15 \%$ were referred on a two week pathway. The presence of dysphagia was the commonest presenting symptom in upper GI malignancy (87\%) but had a poor positive predictive value $(15 \%)$.

Conclusion Nurse led one stop upper GI cancer clinics are safe, allow complete assessment and investigation of patients within 2 week wait (2WW) targets and improve time to test compared to standard clinics. Appropriateness of $2 \mathrm{WW}$ referrals remains low and many cancers are diagnosed outside this pathway. Further use of dedicated nurse led clinics may improve the ability for hospitals to meet service demands.

Disclosure of Interest S. Williams: None Declared, B. Ashall: None Declared, G. Cave: None Declared, A. Bassi: None Declared, P. Flanagan Grant/research support from: Awarded a Shire innovation fund for SpRs.

\section{PTH-071 AUDIT OF SUBSEQUENT OUTCOME IN PATIENTS ADMITTED TO HOSPITAL WITH ALCOHOL USE DISORDER (AUD)}

S Rai* , H Boyce, F Anderson, D Gleeson. Liver Unit, Liver Unit, Sheffield, UK

\subsection{6/gutjnl-2014-307263.517}

Introduction There are limited published data on subsequent outcome of patients admitted acutely to hospital with alcohol use disorders (AUDs), in regard to drinking relapse, hospital readmission and death.

Methods We conducted a prospective audit of 142 patients (105 men), aged (median (range) 46 (23-78) years) admitted with AUDs to a medical admission unit during Oct-Nov 2012 and Jan-March 2013. Information on hospital readmission, AandE attendance and death (from hospital electronic records), and on subsequent alcohol drinking (from records and from telephoning patients) was gathered up to $21 / 05 / 13$. Data were analysed by life-table and Cox regression analysis.

Results Of the 142 patients, 80 (56\%) lived alone and 121 $(85 \%)$ were unemployed. 36 patients $(25 \%)$ had liver disease (Child-Pugh Grade B/C). Of 92 patients with CT or MRI brain scan within 5 years, 49 (53\%) had brain atrophy. 73 patients $(51 \%)$ had another mental health problem (anxiety or depression in 68, schizophrenia in 5). Over the previous year, $71(50 \%)$ had $>1$ previous AUD-related admission, and 24 (17\%) had $>3$ such admissions. Out of 110 patients, $79 \%$ of patients said they intended to stop drinking. Length of stay during index admission was 6 (0-61 days). 51 patients experienced complications, 29 self-discharged early and 18 were verbally \pm physically abusive. 5 patients died during admission, 4 from liver disease.

17 discharged patients were lost to follow up; of the remaining 120, 96 relapsed into drinking, 18 (0-168 days) after discharge. 100-day relapse rate was $78 \%$. When asked the reason for relapse ( $\mathrm{n}=87), 53$ patients cited "no particular reason", 22 depression, 5 a traumatic experience and 4 a celebratory event. Relapse was independently associated with self-discharge after index admission $(\mathrm{p}<0.001)$. 